\title{
Experimental Closed-Loop Excitation of Nonlinear Normal Modes on an Elastic Industrial Robot
}

\author{
Filip Bjelonic $^{1}$, Arne Sachtler ${ }^{1,2}$, Alin Albu-Schäffer ${ }^{1,2}$, and Cosimo Della Santina ${ }^{1,3}$
}

\begin{abstract}
Adding elastic elements to the mechanical structure should enable robots to perform efficient oscillatory tasks. Still, even characterizing natural oscillations in nonlinear systems is a challenge in itself, which nonlinear modal theory promises to solve. Therein eigenmanifolds generalize eigenspaces to mechanical systems with non-Euclidean metrics and thus characterize families of oscillations that are autonomous evolutions of the robot. Eigenmanifolds likewise provide a framework for deriving feedback controllers to excite and sustain these oscillations. Nevertheless, these results have been so far essentially theoretical. They have been applied on relatively low dimensional systems and almost exclusively in simulation. We aim to bridge the theory to the real-world gap with the present work and show that we can excite nonlinear modes in complex systems. To this end, we propose control strategies that can simultaneously stabilize numerically evaluated eigenmanifolds and sustain oscillations in the presence of dissipation. We then focus on the KUKA iiwa with simulated parallel springs as an example of the highly nonlinear and articulated system. We calculate all the nonlinear modes of the system, and we use the proposed strategies to excite the associated natural oscillations.
\end{abstract}

Index Terms-Motion Control; Dynamics; Modeling, Control, and Learning for Soft Robots

\section{INTRODUCTION}

$\mathbf{I}$ $\mathrm{N}$ (articulated) soft robotics, researchers study how introducing elasticity in the mechanical structures of robots [1] may lead to improved performance [2]. The dynamics of these systems are essentially richer than their rigid counterparts, including behaviors like autonomous nonlinear oscillations and chaos. If on the one hand elasticity can generate stabilizing effects, on the other even standard control problems as force and motion control can get more challenging due to reduced control authority [3], [4]. Interestingly, elasticity creates new control opportunities that can be exploited by novel algorithmic solutions.

In this work, we are primarily interested in repetitive motions. In this context, elasticity may help lift efficiency to a higher level. Indeed, energy is not lost during these motions but exchanged between elastic elements and the bodies' velocity. Researchers have considered exploiting elasticity in soft robots to perform locomotion [5], [6] and execute periodic

Manuscript received: September 9, 2021; Revised December 4, 2021; Accepted December 23, 2021. This paper was recommended for publication by the Associate Editor and the Editor C. Gosselin upon evaluation of the reviewers' comments. This work is supported by the ERC advanced grant M-Runners (ID: 835284) and by the EU Project NI (ID: 101016970).

${ }^{1}$ All authors are with the German Aerospace Center (DLR); Institute of Robotics and Mechatronics; Münchener Straße 20, 82234 Weßling, Germany. ${ }^{2}$ A. Sachtler and A. Albu-Schäffer are with the Technical University of Munich (TUM); Department of Informatics; Boltzmannstr. 3, 85748 Garching, Germany. ${ }^{3}$ Cosimo Della Santina is with the Cognitive Robotics Department, TU Delft, Mekelweg 5, 2628 CD Delft, The Netherlands.

(02022 IEEE. Official URL: https://ieeexplore.ieee.org/document/9674820

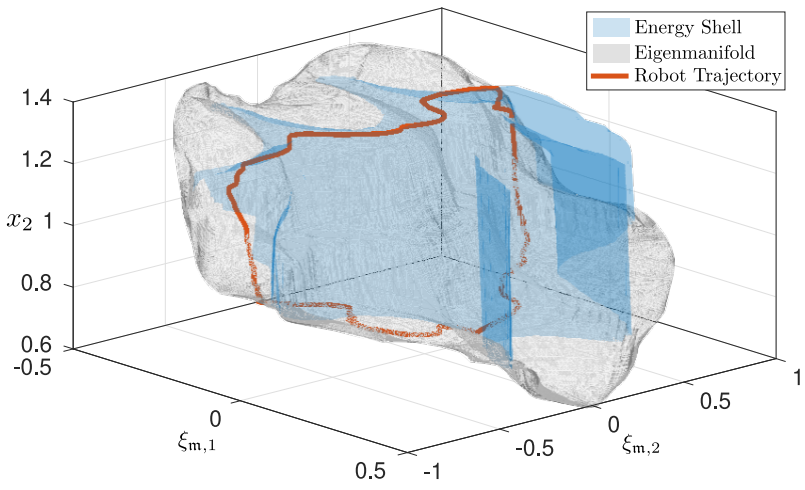

Fig. 1. We show in grey the fourth eigenmanifold $\mathfrak{M}$ of the elastic iiwa projected onto $\left(\xi_{\mathfrak{m}}, x_{2}\right) \in \mathbb{R}^{3}$, where $\xi_{\mathfrak{m}}$ are the modal coordinates. The surface is a section of the energy shell $\mathcal{E}(\bar{E})$, and it is shown only on one side of the eigenmanifold not to overload the figure. The proposed control strategy regulates nonlinear modes by simultaneously stabilizing $\mathfrak{M}$ and $\mathcal{E}$. The smooth robot trajectory in orange comes from multiple experimental cycles, and it correctly sits close to the intersection of the two surfaces.

tasks in an industrial context [7]-[11]. Consequently, many algorithms have been recently proposed that stabilize nonlinear oscillations in mechanical systems. In this context, virtual holonomic constraints have generated sound theoretical results [12]-[14]. Alternatively, transverse feedback linearization [15], energy shaping [16], Floquet theory [17], immersion and invariance [18] have all been considered. Finally, bio-inspired solution combined with engineering intuition yield promising but task- and platform-specific results [19], [20]. But all these techniques are not designed to minimize the control effort, and as such, they involve some form of steady-state model cancellation.

As an alternative, with a series of recent works [21][24], we have proposed sacrificing versatility for efficiency. Instead of prescribing some oscillatory behavior to the system - not matched to the physics of the system - we propose to start by characterizing what the robot is good at utilizing nonlinear modal theory. Nonlinear modes provide an extension of the linear modal theory to nonlinear systems [23], [25]. Eigenvectors become curves, eigenspaces become eigenmanifolds, and sinusoidal oscillations become nonlinear periodic orbits. Eigenmanifolds are collections of self-similar periodic orbits that the mechanical system can perform as autonomous evolution. Therefore, they can be seen as a way of characterizing all the regular oscillatory behaviors of (soft) robots. Once the eigenmanifolds of the robot have been identified, controllers can be devised to excite and stabilize the associated oscillations [21], [22]. This can be achieved by simultaneously transforming an eigenmanifold into an attractor, and regulating the total energy of the system (Fig. 11). The results are stable hyper-efficient natural oscillations. However, these techniques 
have had a primarily theoretical development and were validated on simple systems and exclusively in simulation - except for the preliminary work [26].

This paper aims to finally provide a long-awaited solid experimental validation to this otherwise theoretical framework, even if preliminary. As we will discuss later in this paper, this also requires developing new control strategies that - even if based on the same philosophy of [21], [22] can deal with challenges that ideal theoretical formulations would neglect. We compute and report a representation of the eigenmanifolds and evaluate the control approaches on an industrial manipulator with seven degrees of freedom (elastic KUKA iiwa robot). We demonstrate the excitation of various nonlinear modes from six out of the seven eigenmanifolds.

\section{BACKGROUND AND NOTATION ON NONLINEAR NORMAL MODES}

The theory of nonlinear normal modes in conservative mechanical systems is the base upon which we build the results proposed in this paper. We report here an introduction to the topics that will be necessarily succinct and informal for the sake of space. The interested reader can refer to [23] for a general introduction to the topic.

\section{A. General Notation}

Consider a nonlinear mechanical system governed by the multi-body dynamics equation

$$
M(x) \ddot{x}+C(x, \dot{x}) \dot{x}+\frac{\partial V}{\partial x}(x)=\tau,
$$

where $x \in \mathbb{R}^{n}$ are Lagrangian coordinates (e.g., joint variables), $\dot{x}$ and $\ddot{x}$ their first and second time derivatives. The inertia matrix $M(x) \in \mathbb{R}^{n \times n}$ is assumed positive definite, and $C(x, \dot{x}) \in \mathbb{R}^{n \times n}$ combines Coriolis and centrifugal terms. Finally, $V: \mathbb{R}^{n} \rightarrow \mathbb{R}$ is the potential field induced by, e.g., gravity and elastic actions. The total energy $E$ of the system (1) is

$$
E(x, \dot{x})=\frac{1}{2} \dot{x}^{\top} M(x) \dot{x}+V(x) .
$$

We assume that there is at least one isolated $x_{\text {eq }}$ such that it is a local minimum of $V$, or equivalently a stable equilibrium of (1). We assume the level curves of $V$ are closed in a large enough neighborhood of $x_{\mathrm{eq}}$. The vector norm is defined as $\|x\|_{M}=\sqrt{x^{\top} M x}$.

\section{B. Modes}

The spectral analysis of $S^{-1 / 2} M\left(x_{\mathrm{eq}}\right) S^{1 / 2}$ yields $n$ independent eigenvectors, with $S$ being the stiffness matrix at the equilibrium defined as the Hessian of $V$. Each eigenvector $c$ leads to a separate two-dimensional eigenspace $E S=$ $\operatorname{Span}\{(c, 0),(0, c)\}$. This is an invariant space for the linear approximation of the robot, with all evolutions being regular sinusoidal oscillations. However, this analysis holds only under the small-oscillations hypothesis.

The eigenmanifold theory aims to extend eigenspaces outside small oscillations to characterize families of largeamplitude nonlinear oscillations. Therefore, we define an eigenmanifold $\mathfrak{M} \subset \mathbb{R}^{2 n}$ as a two-dimensional invariant submanifold of the state space. The invariance is here defined w.r.t. the dynamics (1), and thus outside any small oscillation regime. There are at least $n$ distinct eigenmanifolds associated with an equilibrium $x_{\text {eq }}$, and each one of them is tangent to a different linear eigenspace $E S$. An eigenmanifold is a continuous collection of periodic orbits (eigenmodes from now on), each of which can be univocally identified by its constant (pseudo-)energy. For small energies, these orbits will resemble the ones predicted by the linear analysis. In other words, every initial condition $(x(0), \dot{x}(0)) \in \mathfrak{M}$ leads to a periodic orbit whenever $\tau \equiv 0$, and thus the forward evolution stays on $\mathfrak{M}$ without applying torques.

Given an eigenmanifold $\mathfrak{M}$, we can identify two functions $(X, \dot{X}): \mathbb{R}^{2} \rightarrow \mathbb{R}^{n} \times \mathbb{R}^{n}$ such that we can define the manifolds as the image of these maps, i.e.,

$$
\mathfrak{M}=\left\{(x, \dot{x}) \in \mathbb{R}^{2 n} \mid \exists \xi_{\mathfrak{m}} \in \mathbb{R}^{2} \text { s.t. }(x, \dot{x})=\left(X\left(\xi_{\mathfrak{m}}\right), \dot{X}\left(\xi_{\mathfrak{m}}\right)\right)\right\} .
$$

The two-dimensional variable $\xi_{\mathfrak{m}}$ is a parameterization of the eigenmanifold, and $(X, \dot{X})$ is the natural embedding in the associated coordinates. This paper will propose algorithms that do not need to explicitly calculate these quantities since they are not easily obtainable for high-dimensional systems.

\section{Control Strategy}

The goal of this section is to introduce a control architecture that excites and stabilizes a large palette of eigenmodes from a complex mechanical system, such as the elastic industrial manipulator that we consider in the experimental section.

We base this architecture on the approach that some of the authors of the present paper have proposed in [21], [22]. This framework builds upon the fact that the trajectory spanned by an eigenmode is always coincident with $\mathfrak{M} \cap \mathcal{E}(\bar{E})$, where

$$
\mathcal{E}(\bar{E})=\left\{(x, \dot{x}) \in \mathbb{R}^{2 n} \mid E(x, \dot{x})=\bar{E}\right\}
$$

is the energy shell of energy $\bar{E}$. Note that for a conservative system this is a $(2 n-1)$-dimensional invariant manifold. Thus, $\mathfrak{M} \cap \mathcal{E}(\bar{E})$ has correctly dimension 1 (single orbit). Fig. 1 reports an example of these two manifolds, together with an experimental oscillation obtained as their intersection.

Based on this intuition, we split our controller into

$$
\tau(x, \dot{x})=\tau_{\mathrm{M}}(x, \dot{x} ; \mathfrak{M})+\tau_{\mathrm{E}}(x, \dot{x} ; \bar{E}),
$$

such that

(i) $\tau_{\mathrm{M}}$ acts as an eigenmanifold stabilizer, producing closedloop trajectories such that $(x(\infty), \dot{x}(\infty)) \in \mathfrak{M}$ or alternatively $(x(\infty), \dot{x}(\infty))=\left(X\left(\xi_{\mathfrak{m}}\right), \dot{X}\left(\xi_{\mathfrak{m}}\right)\right)$ for some $\xi_{\mathfrak{m}} \in \mathbb{R}^{2}$

(ii) $\tau_{\mathrm{E}}$ acts as an energy regulator, thus implementing a closed-loop energy evolution such that $E(\infty)=\bar{E}$.

If both controllers succeed in their tasks, then $(x, \dot{x})$ converges to a modal orbit and $\tau \rightarrow 0$. This section aims at introducing new controllers $\tau_{\mathrm{M}}$ and $\tau_{\mathrm{E}}$ achieving their respective goals under realistic hypotheses. The novel control architecture that we designed is visualized as a block diagram in Fig. 2 Here, we already show the system that will be used in the experimental section. 


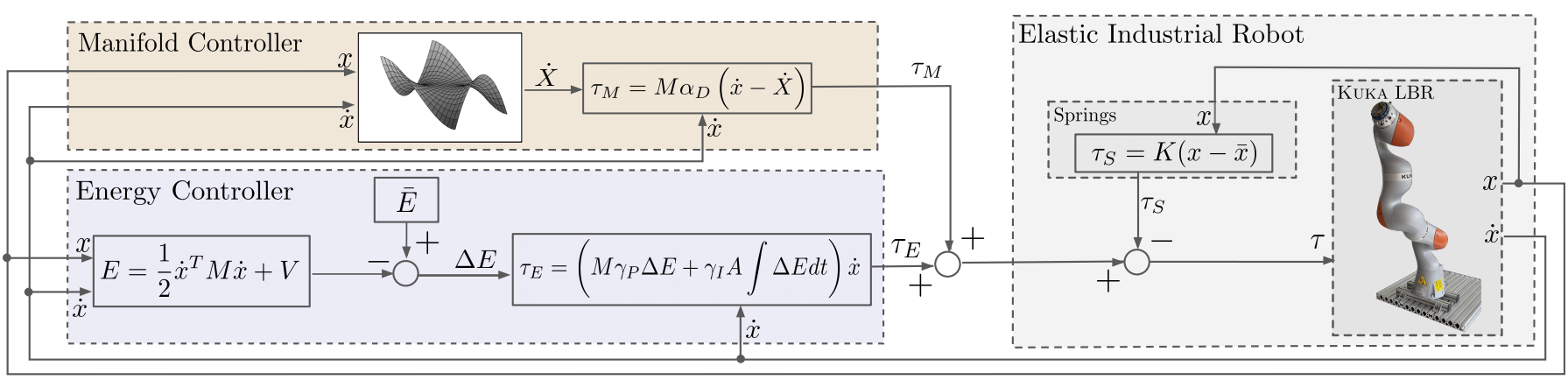

Fig. 2. Block diagram of the control architecture. Both control loops are reported in the figure: the manifold controller $\tau_{\mathrm{M}}$ that stabilizes the eigenmanifold, and the energy controller $\tau_{\mathrm{E}}$ that regulates the energy $E$ to the desired reference $\bar{E}$. Together, they excite and sustain the nonlinear modes whose trajectory is $\mathfrak{M} \cap \mathcal{E}(\bar{E})$. The physical system used in the experimental validation is a KUKA iiwa with simulated parallel springs implemented by $\tau_{\mathrm{S}}$. Note however that the proposed control strategy can be applied to any articulated soft robot with physical parallel elasticity.

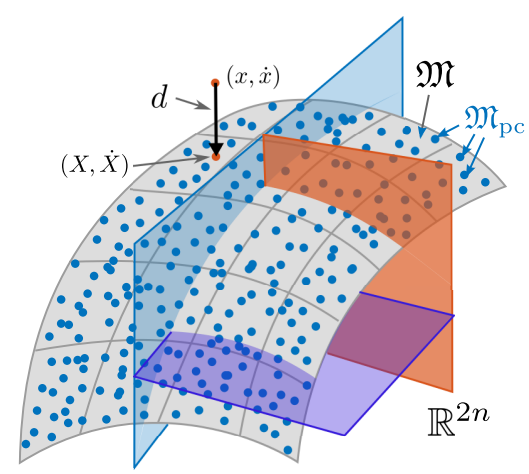

(a) Point Cloud Representation

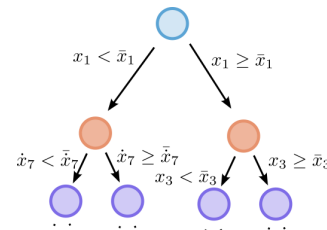

(b) Tree Data Structure

Fig. 3. Coordinate expression of the eigenmanifold $\mathfrak{M}$ with dots inside visualizing the pointcloud; and a sketch of the tree data structure for fast closest-point queries.

\section{A. Eigenmanifold Stabilization}

The strategy for the eigenmanifold stabilization that we have proposed in previous work [21] does not trivially generalize to high dimensional systems. Indeed, it requires a closed-form expression of $(X, \dot{X})$ which is hard to obtain in practice. Also, that strategy is intrinsically local since it relies on projection onto the tangent eigenspace $E S$. Here, we propose an eigenmanifold stabilization strategy that is globally well defined, does not rely on any closed-form parameterization and is easy enough to implement in a realistic scenario.

First, we do not explicitly create a parameterization of the eigenmanifold but rather represent it by a set of points. We employ a shooting method that starts from the equilibrium, points in the direction of the eigenvector, and from there locally searches for modes of increasing energy - similarly as discussed in [23, Sec. 8]. We simulate each of the modal oscillations so discovered by forward-integration of (1). We stop the simulation after one complete oscillation. The collection of all these samples yields a point cloud $\mathfrak{M}_{\mathrm{pc}}$, which serves as a numerical and discrete approximation of $\mathfrak{M}$ (cp. Fig. 3).

In preparation to define a controller, we design a retraction of any state $(x, \dot{x})$ onto the eigenmanifold using its point cloud representation by solving the following discrete optimization

$$
\left(X\left(\xi_{\mathfrak{m}}\right), \dot{X}\left(\xi_{\mathfrak{m}}\right)\right)=\underset{(\tilde{x}, \tilde{x}) \in \mathfrak{M}_{\mathrm{pc}}}{\arg \min }\left\|\left[\begin{array}{c}
x \\
\dot{x}
\end{array}\right]-\left[\begin{array}{c}
\tilde{x} \\
\dot{x}
\end{array}\right]\right\|_{2}^{2} .
$$

Given a state vector $(x, \dot{x})$, this will return the point in $\mathfrak{M}_{\mathrm{pc}}$ closest to $(x, \dot{x})$ w.r.t. Euclidean distance. Note that we do not evaluate $\xi_{\mathfrak{m}}$ explicitly, but directly the element in the eigenmanifold as appearing embedded in $\mathbb{R}^{2 n}$. This way, we are neither constrained by the possible locality of the parameterization $(X, \dot{X})$ nor by the implicit dependency of $\xi_{\mathfrak{m}}$ in 6 .

The naïve approach to solving (6) is to compare each point in $\mathfrak{M}_{\mathrm{pc}}$ to the query point $(x, \dot{x})$. The complexity of this algorithm is $\mathcal{O}(m)$, with $m$ being the number of points stored in $\mathfrak{M}_{\text {pc }}$. For good enough approximations of $\mathfrak{M}$, this approach becomes very soon computationally intractable. We propose the following k-d tree strategy [27] as an alternative. First, we pre-compute a tree data structure on top of the point cloud. Each node of the tree represents a cut of the state space by a hyperplane (cf. Fig. 3). The k-d tree algorithm selects the dimension and the threshold value along which the cut is performed for each node such that the tree implements a fast closest-point query. Given a query point $(x, \dot{x})$ the tree is traversed and the leaf node corresponds to the closest point. This strategy has query times in $\mathcal{O}(\log m)$ and, thus, makes it feasible to implement the retraction (6) within a controller cycle. This strategy directly generalizes to generic distances used in (6) in place of the Euclidean one. For example, the distance induced by the Sasaki metric [28] would be a more physically correct choice, however hard to compute in practice.

Using (6) we can define a distance vector to the eigenmanifold, and thus propose the following $D$-like controller

$$
\tau_{\mathrm{M}}\left(x, \dot{x} ; \mathfrak{M}_{\mathrm{pc}}\right)=\alpha_{\mathrm{D}} M(x)\left(\dot{x}-\dot{X}\left(\xi_{\mathfrak{m}}\right)\right) .
$$

Here, $\alpha_{\mathrm{D}}>0$ is a scalar gain, and $\dot{X}\left(\xi_{\mathfrak{m}}\right)$ is the vector of joint velocities of the closest point to $(x, \dot{x})$ in $\mathfrak{M}_{\mathrm{pc}}$ in an Euclidean sense - found as a solution of (6). It is worth stressing that this strategy always pushes the robot towards the manifold, no matter how distant the state is from it. Given the experimental nature of this work, we are not interested here in formally assessing the stability properties of this strategy. It is however worth mentioning that neither in simulation, nor with experiments, we ever experienced a condition in which this controller has not converged to a neighborhood of $\mathfrak{M}$. 


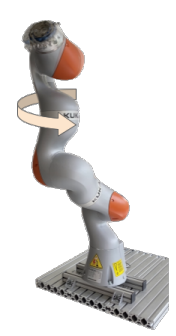

(a) Mode 1

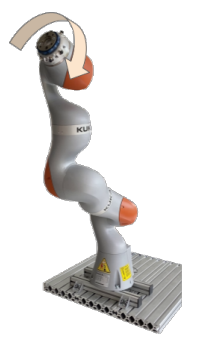

(b) Mode 2

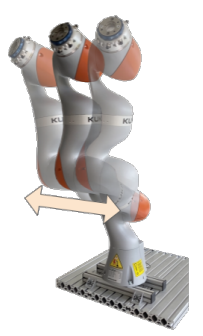

(c) Mode 3

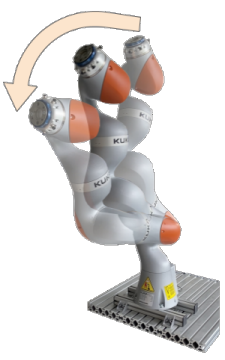

(d) Mode 4

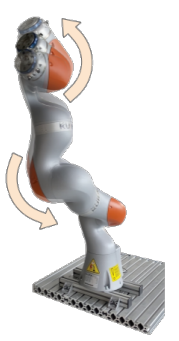

(e) Mode 5

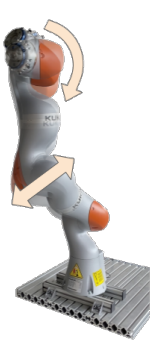

(f) Mode 6

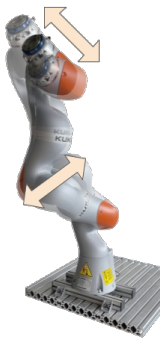

(g) Mode 7

Fig. 4. Identified nonlinear normal modes of the KUKA iiwa. For each mode, an oscillation for one energy level is sketched. The arrows indicate the main motion of the mode.
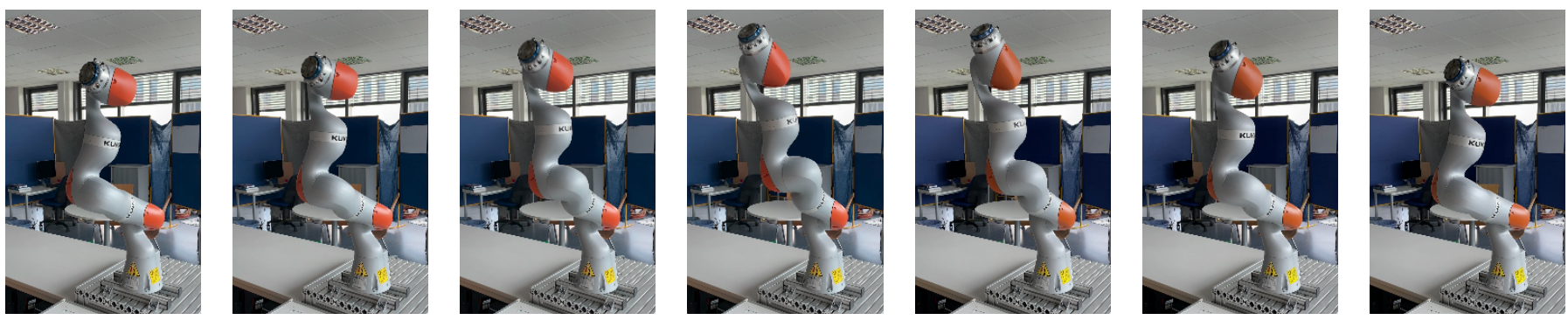

Fig. 5. Stills of a single period of the steady-state oscillation associated to eigenmanifold 7 , as obtained during the experiments in IV-C Time flows from left to right and the period of this oscillation is $1 \mathrm{~s}$.

\section{B. Energy Regulation}

Eigenmanifold theory is developed under the assumption that the system is conservative. This is a hypothesis that it is clearly not going to be fulfilled in the practice, since dissipation is an unavoidable aspect of any real scenario. Here, we introduce a heuristic mechanism of damping compensation for the first time in the eigenmanifold framework.

We start by reformulating the dynamics as $M(x) \ddot{x}+$ $C(x, \dot{x}) \dot{x}+D(x) \dot{x}+\frac{\partial V}{\partial x}(x)=\tau$, where $D \succ 0$ is the damping matrix. We propose the following energy regulator

$$
\begin{aligned}
\tau_{\mathrm{E}}(x, \dot{x} ; \bar{E}) & =\gamma_{\mathrm{P}} M(x)(\bar{E}-E(x, \dot{x})) \dot{x} \\
& +\gamma_{\mathrm{I}} A(x)\left(\int(\bar{E}-E(x, \dot{x})) \mathrm{d} t\right) \dot{x} .
\end{aligned}
$$

Here, $\gamma_{\mathrm{P}}, \gamma_{\mathrm{I}}>0$ are scalar controller gains, $E(x, \dot{x})$ is the energy as defined in (2), and $A$ is a positive definite matrix.

To assess the asymptotic behavior of the closed-loop, we first evaluate the rate of change of the energy, which is

$$
\begin{aligned}
\dot{E} & =\dot{x}^{\top} M(x) \ddot{x}+\frac{1}{2} \dot{x}^{\top} \dot{M}(x) \dot{x}+\frac{\partial V(x)}{\partial x} \dot{x} \\
& =-\dot{x}^{\top} D \dot{x}+\dot{x}^{\top} \tau_{E} .
\end{aligned}
$$

Plugging (8) into (9), and defining $\Delta=\bar{E}-E$ yields the closed-loop energy evolution

$$
\dot{\Delta}=-\gamma_{\mathrm{P}}\|\dot{x}\|_{M}^{2} \Delta-\|\dot{x}\|_{A}^{2}\left(\gamma_{\mathrm{I}} \int \Delta \mathrm{d} t-\beta\right) \dot{x} .
$$

Note that we have hypothesized that $\beta \in \mathbb{R}$ exists such that $D=\beta A$. This is clearly a strong assumption, but nevertheless, we have observed that this controller gives robust results in the practice. Eq. (10) is a second-order scalar and linear system, with time-varying positive gains $\|\dot{x}\|_{M}^{2},\|\dot{x}\|_{A}^{2}$ that annihilate only at isolated points in time. As such, it converges globally (but not exponentially) to $\Delta$. Thus $\tau_{\mathrm{E}}$ implements a perfect compensation of the damping $D \dot{x}$, with its integral term will converge to an estimate $\beta$, under the stringent hypothesis that $D=\beta A$. To conclude, it should be noted that the convergence of this energy regulator does not automatically imply that the full controller (5) converges to a stable oscillation. See [22] for more details on this topic.

\section{EXPERIMENTAL INVESTIGATION}

We conducted four sets of tests to assess the proposed controller's effectiveness, robustness, and efficiency. The first test shows that the control architecture can excite and sustain the desired mode - i.e., stabilize the eigenmanifold, and regulate the energy level. The second test looks at disturbance rejection. Afterwards, we test the controller's capabilities in dynamically switching from one mode to another in the third experiment. We test changes of eigenmanifold $\mathfrak{M}$ as well as changes of energy shell $\mathcal{E}$. The fourth experiment looks more closely at changes in the desired energy and the oscillation amplitude with it. Note that we aim here at exciting periodic orbits that are, on average, close to the theoretical manifolds. In most plots, we report both the evolution in light grey and its average over one period. Control gains are as reported in Table I. Although most of the discussion is devoted to robustness and effectiveness, we devote a subsection at the end to report about efficiency.

\section{A. Robotic System}

The goal of this section is to show that the proposed control architecture can excite modal oscillations in an industrial robot. Unfortunately, we did not have any highly articulated elastic system available. As an alternative, we decided to use 

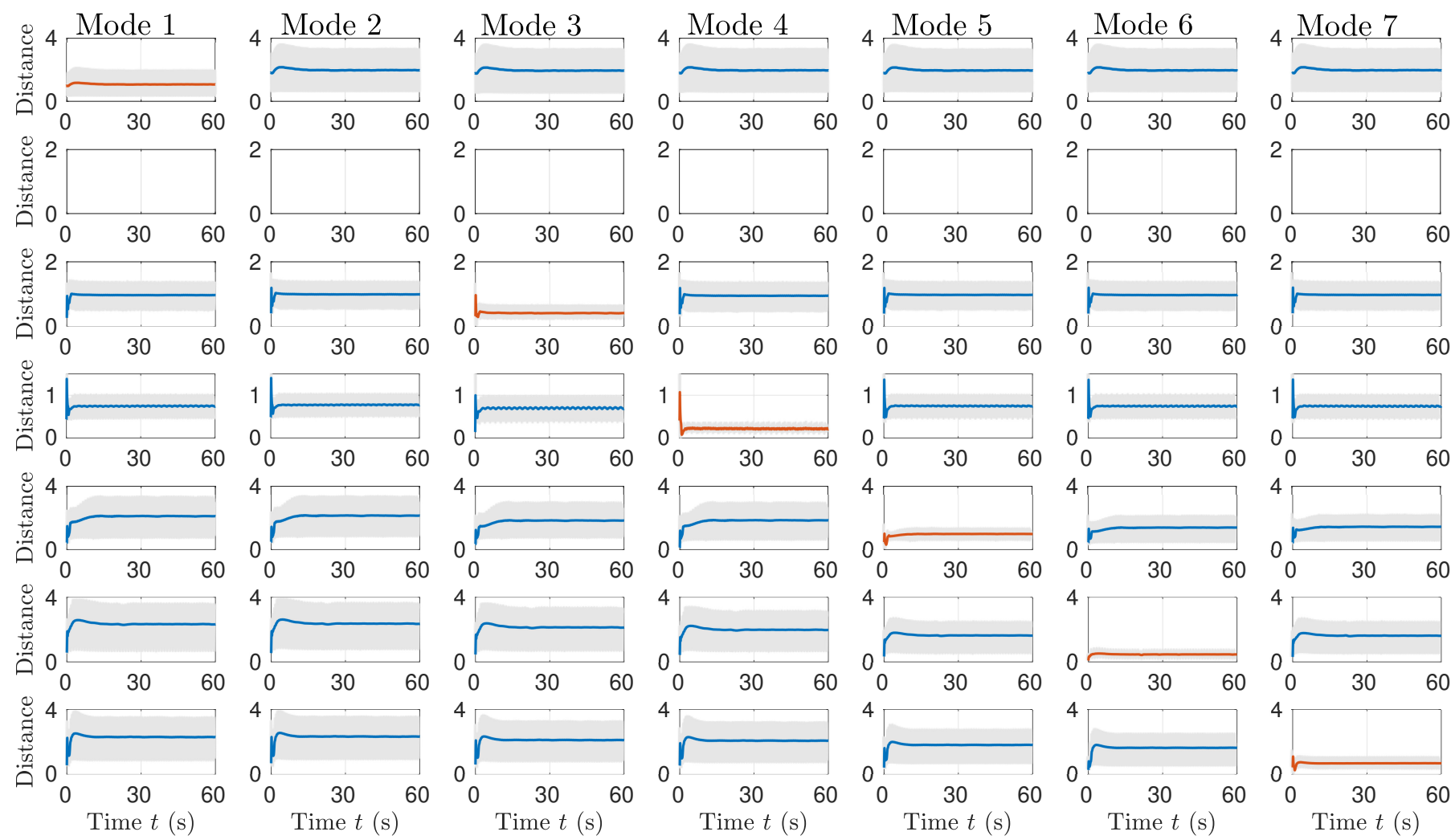

Fig. 6. The eigenmanifold controller successfully minimizes the distance of the state to the eigenmanifold. Each row shows the excitation experiment for each eigenmode. Since we have seven eigenmanifolds, each column shows the distances with respect to the specific eigenmode. As a result, the plots on the diagonal (orange lines) show always the smallest in distance.
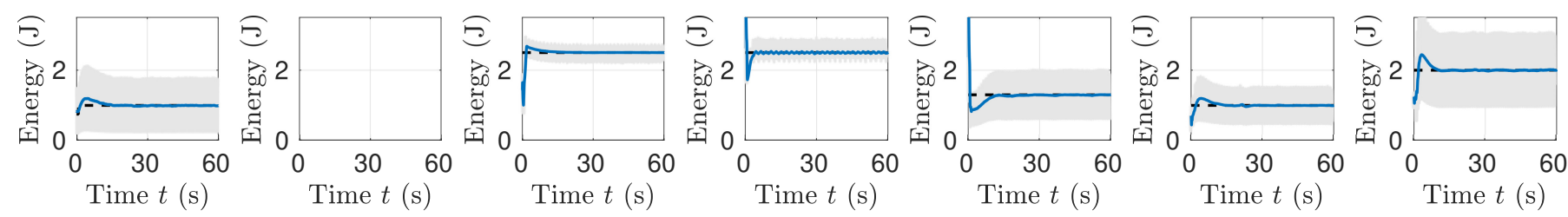

Fig. 7. Energy evolution during the excitation experiment (cf. Fig. 6). The combined controller is not only capable of minimizing the distance to the manifold but is also able to simultaneously regulating the desired energy level (dashed black line).

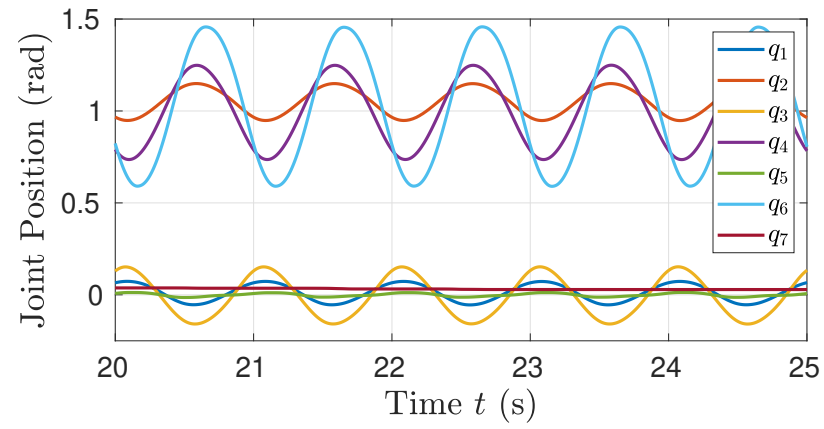

Fig. 8. This figure illustrates the joint configuration over time for five consecutive oscillations of eigenmode 7 . The corresponding sequence of stills is shown in Fig. 5

the KUKA LBR iiwa 7 R800 as seen in Fig. 4 A low level closed-loop $\left(\tau_{\mathrm{S}}\right.$ in figure) simulates the presence of parallel joint-level springs with potential $(x-\bar{x})^{\top} K(x-\bar{x}) / 2$, with $K=\operatorname{diag}([50,50,50,50,50,5,1]) \mathrm{N} \mathrm{m} \mathrm{rad}^{-1}$ being the stiffness matrix and $\bar{x}=[0,1,0,1,0,1,0]^{\top} \mathrm{rad}$ being the

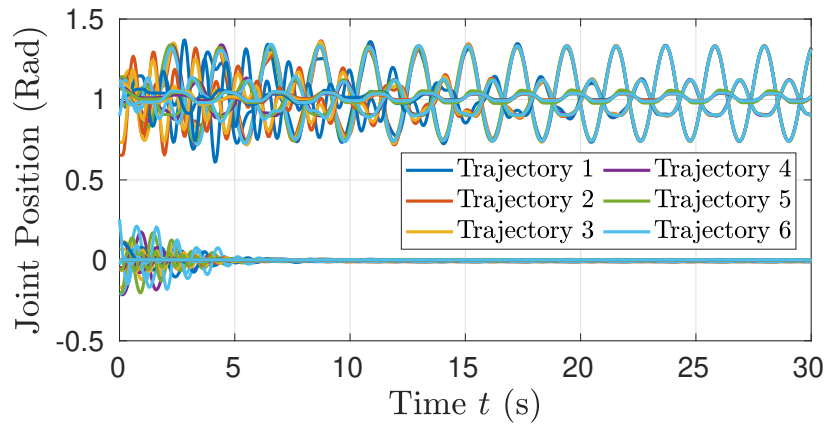

Fig. 9. This figure shows the trajectories of 6 different initial positions of the robot with the control goal being mode 4 and desired energy of $2.5 \mathrm{~J}$. Each color corresponds to 7 lines, one line per joint position. After 10s, the lines start to overlap, which means that the controller is able to push the system onto the desired eigenmode and energy level for a variety of starting configurations.

equilibrium positions of the springs. The $A$ matrix was set to be $M(x)$. The update time of the controller is $5 \mathrm{~ms}$. The 
joint velocity is computed by finite difference. The equations of motion are calculated using a state of the art dynamics algorithm and the robot URDF-File provided in [29]. For the computation, we assumed a conservative mechanical system with parallel springs in its joints. The result is the dynamics equation as seen in (1).

The KUKA robot has a partial gravity compensation implemented as built-in feature that cannot be disabled. We could identify the residual gravity acceleration to be approximately $\tilde{g}=0.275 \mathrm{~m} \mathrm{~s}^{-2}$. Moreover, a low-level compensation of friction is present as well - which not only does not wholly compensate for dissipation, but at times it overcompensates. These various effects build up considerable uncertainty, which makes the control challenge especially hard. Still, the proposed control strategy works robustly, always converging to steadystate oscillations - as discussed below. For example, due to friction overcompensation, the energy may even diverge in open loop. The proposed energy controller $\tau_{\mathrm{E}}$ appears robust enough to converge to a dissipation action rather than to an energy injection when this is needed to keep the right energy balance.

\section{B. Nonlinear Eigenmodes of the Elastic LBR}

This system has seven independent eigenmanifolds, one for each linear mode. Calculation of all modes with the strategy discussed above took 2.71 hours on an Intel Core i5-5200U $\mathrm{CPU}$. Note that these are offline calculations that are required only once. An example of modal oscillation - as excited by our closed-loop strategy - for each eigenmanifold can be seen in Figs. 4, 5 and the supplementary video. The first four modes are dominated by oscillations of a single joint - numbers 5 , 7,1 , and 2 , respectively. The remaining three modes involve coordinated patterns of oscillation of several joints.

\section{Exciting Eigenmodes}

We report here on the excitation of the eigenmode of maximum energy (and amplitude) allowed for each of the seven eigenmanifolds. We have computed $\mathfrak{M}_{\mathrm{pc}}$ up to $10 \mathrm{~J}$. However, LBR's joint limits did not allow for testing up to this energy. We report the actual maximum energies that the hardware can accept in Tab. I] We initialize the robot at a point close to the eigenmanifold, and let the oscillation continue for $60 \mathrm{~s}$. Fig. 6 shows in each line the result of this experiment

TABLE I

CONTROLLER GAINS AND MAXIMUM ENERGY

\begin{tabular}{c|c|c|c|c|c|c|c} 
Mode & 1 & 2 & 3 & 4 & 5 & 6 & 7 \\
\hline$\alpha_{D}$ in $\frac{\mathrm{s}^{2}}{\mathrm{~kg} \mathrm{~m} \mathrm{rad}}$ & -3 & - & -7 & -2 & -14 & -13 & -14 \\
\hline$\gamma_{\mathrm{P}}$ in $\frac{\mathrm{s}}{\mathrm{kg} \mathrm{rad}}$ & 10 & - & 5 & 1 & 10 & 5 & 4 \\
\hline$\gamma_{\mathrm{I}}$ in $\frac{1}{\mathrm{~kg} \mathrm{rad}}$ & 1 & - & 1 & 1 & 1 & 1 & 1 \\
\hline max Energy in J & 1.0 & - & 2.5 & 2.5 & 1.3 & 1.0 & 2.0
\end{tabular}

TABLE II

PERFORMANCE FOR EXCITING EIGENMODES

\begin{tabular}{c|c|c|c|c|c|c|c} 
Eigenmode & 1 & 2 & 3 & 4 & 5 & 6 & 7 \\
\hline Distance & 1.1 & - & 0.41 & 0.23 & 0.98 & 0.46 & 0.68 \\
\hline$\Delta$ Energy J & 0.0 & - & 0.0 & 0.0 & 0.0 & 0.0 & 0.0 \\
\hline Overshoot J & 0.19 & - & 0.17 & 0.0 & 0.0 & 0.2 & 0.44 \\
\hline Settling Time s & 13 & - & 7.5 & 2.6 & 9.9 & 12 & 8.4
\end{tabular}

TABLE III

EIGENFREQUENCIES OF THE MODES

\begin{tabular}{c|c|c|c|c|c|c|c} 
Mode & 1 & 2 & 3 & 4 & 5 & 6 & 7 \\
\hline $\mathrm{f}$ in $\mathrm{Hz}(\mathrm{sim})$ & 5.3 & 3.0 & 0.68 & 0.44 & 2.0 & 1.7 & 1.9 \\
\hline $\mathrm{f}$ in $\mathrm{Hz}(\mathrm{real})$ & 1.8 & - & 0.64 & 0.47 & 1.0 & 0.90 & 1.0
\end{tabular}

for each eigenmode. The columns refer to the distance to each manifold evolution at the desired energy level for the specific experiment. Our controller failed to excite mode 2 which would have involved mostly an oscillation on the last joint. This failure is due to the high level of static friction affecting this joint in our experimental platform, together with the low inertia of the corresponding link. For all other modes, the manifold controller $\tau_{\mathrm{M}}$ successfully reduces the distance to the selected eigenmanifold, as proven by the fact that the diagonal elements are the smallest. Fig. 7 reports the energy evolution together with its reference as a black dashed line. Thus, also the energy regulator $\tau_{\mathrm{E}}$ successfully converges to the desired behavior. Figs. 5.8 show an example of steadystate oscillations achieved in this experiment, corresponding to eigenmanifold 7.

We summarize some performance metrics in Tab. II] whose first two rows report the steady-state Euclidean distance to the eigenmanifold and the energy error respectively. Row 3 reports the maximum energy overshoot, and row 4 the $2 \%$ settling time. Tab. III reports the frequencies for the calculated modes in simulation and the once measured during these experiments. Interestingly, the latter are always smaller than the former. This is coherent with linear modal analysis, that shows that adding damping reduces the natural frequency of oscillation of a linear mechanical system. This effect is especially visible for modes $1,5,6$, and 7 , which are the ones involving larger oscillations of the last three joints of the robot. There, the friction torque becomes a dominant factor.

To test the controller's capabilities of working from different starting conditions, Fig. 9 shows the system's trajectory in joint space for controlling eigenmode 4 with six random initial conditions. A total of 20 initial conditions have been tested with similar results. We do not report them here for the sake of clarity. In all of the tested cases, the controller successfully converged towards the desired mode.

\section{Rejection of Disturbances and Uncertainties}

We test here the ability of the closed-loop to reject disturbances. We disturbed oscillations by pushing the system at points corresponding to joints $2,3,4,5$ and 6 . We have repeated the experiment for each mode except the second (see IV-B]. In each experiment, the controller smoothly guided the evolution back to its original oscillation. Due to space limitations, we could report here only one of these experiments in Fig. 10. Here we push the robot at the joint 4 in two different directions, and sideways at joint 6 . Fig. 10d shows that both control goals are always restored: the system goes back to the eigenmanifold, and to the desired energy level of $1 \mathrm{~J}$. Similar performance are achieved for the experiments with the other modes.

Additionally, we performed experiments with an additional payload connected to the end effector of our KUKA robot. Its weight is $0.38 \mathrm{~kg}$ (about $10 \%$ the mass of a link) is unknown to the controller, and as such serves as model uncertainty. The 

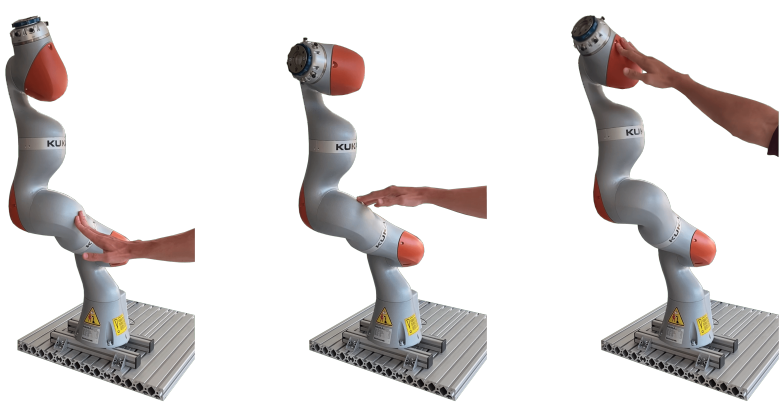

(a) Lateral, Joint 4

(b) Anterior, Joint 4

(c) Lateral, Joint 6

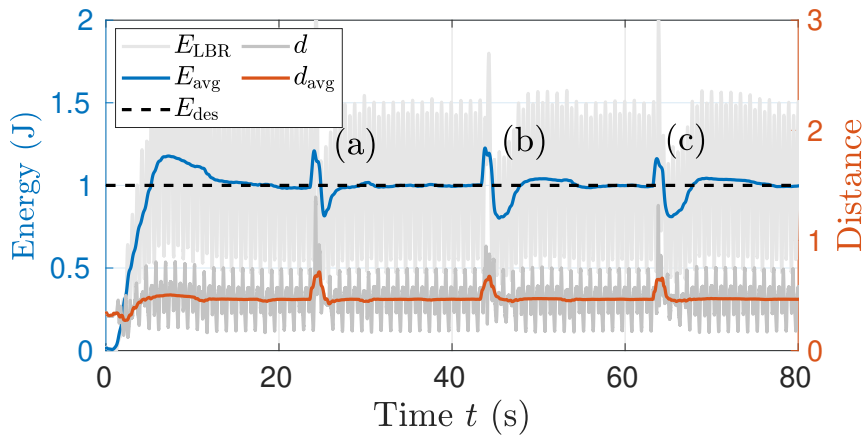

(d) Energy and distance plot

Fig. 10. This is an example of disturbing manually a mode from section IV-D At time $24 s$, we push the system as shown in Fig. 10a followed by one hit like Fig. 10b at $44 s$ and one last disturbance at $64 s$. Below that in Fig. $10 \mathrm{~d}$ is the evolution of the energy (blue), the distance to the eigenmanifold (orange) and the desired energy (dashed black line) displayed.

controller still managed to excite all modes successfully. We observe that the resulting frequency of the oscillation drops slightly, e.g. $2 \%$ for mode 6 .

\section{E. Changing Modes}

In this experiment, we initialize the controller to stabilize at the sixth eigenmanifold. After $10 \mathrm{~s}$, when the robot reaches the steady-state oscillation, we change the desired eigenmanifold to the seventh. We also increase the desired energy $\bar{E}$ from $1 \mathrm{~J}$ to $2 \mathrm{~J}$. The evolution of the distance to the two eigenmanifolds is reported in Fig. 11, which clearly shows the switch from one manifold to the other. This highlights that, without further modification, the controller can change dynamically from one mode to another without divergence or failure.

\section{F. Increasing Energy}

Our last experiment focuses on the regulation of the energy, while $\tau_{\mathrm{M}}$ keeps regulating mode 3 . Fig. 12 shows two energy jumps of amplitude $0.75 \mathrm{~J}$ every $10 \mathrm{~s}$. The controller $\tau_{\mathrm{E}}$ drives the system to the new energy levels in a few seconds. A finer set of increments is shown in Fig. 13 The desired energy starts at $1 \mathrm{~J}$ and increases every $2 \mathrm{~s}$ by $0.1 \mathrm{~J}$ up to the maximum tested energy of $2.5 \mathrm{~J}$.

\section{G. Efficiency and Energy Consumption}

Although this has not been an explicit focus of this paper, one of the main goals of controlling eigenmanifolds

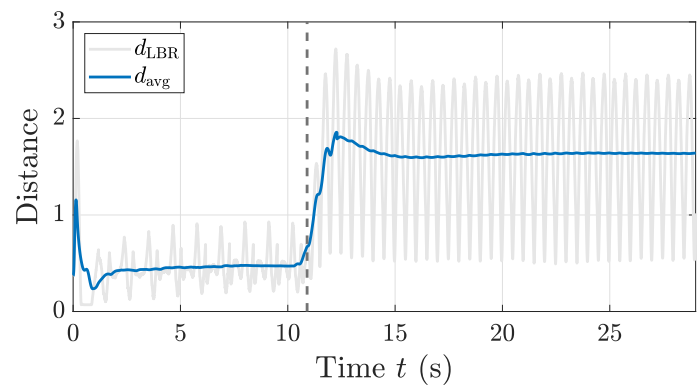

(a) Distance to eigenmode evolution 6.

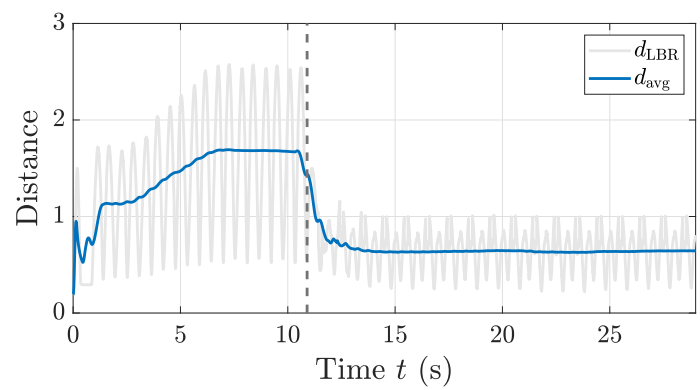

(b) Distance to eigenmode evolution 7.

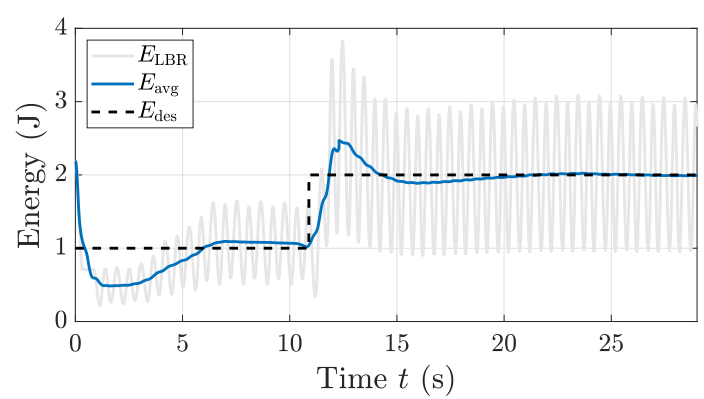

(c) Energy plot.

Fig. 11. These plots indicate the dynamic capabilities of the controller switching from one mode (6) to another mode (7). Pane (a) and (b) show the distance to the manifold respectively. It shows, that the proposed controller is capable of changing modes robustly, while still reaching the desired energy level (dashed black line) in (c) after a few seconds.

is to implement hyper-efficient oscillations. Indeed, modes are defined such that they do not require any torques to be sustained if there is no friction. We first calculate the energy consumption of our system for the rigid and the simulated elastic version. The energy consumption can be estimated as $E_{\text {rigid }}=\sum_{i=1}^{n}\left(\tau_{\mathrm{M}}\left(t_{i}\right)+\tau_{\mathrm{E}}\left(t_{i}\right)-K\left(x\left(t_{i}\right)-\bar{x}\right)\right) \Delta x\left(t_{i}\right)$ and $E_{\mathrm{soft}}=\sum_{i=1}^{n}\left(\tau_{\mathrm{M}}\left(t_{i}\right)+\tau_{\mathrm{E}}\left(t_{i}\right)\right) \Delta x\left(t_{i}\right)$. The energy consumption for the experiments in Sec. IV-C are reported in the first and second row of Table IV] as well as the ratio of energies $E_{\text {soft }} / E_{\text {rigid }}$ in the third row. The reduction of energy consumption due to elastic elements is between $56 \%$ and $94 \%$. Clearly, results should be taken with care and further in-depth efficiency studies are needed to fully characterize the efficiency of the eigenmanifold framework.

Additionally, we report the ratio of energies $E_{\text {soft }} / E_{\mathrm{tt}}$ between our controller and a state of the art PD+ trajectory tracking controller to follow our eigenmodes from the simulation. Even though the trajectory tracking controller has access to the springs and the eigenmanifold, our controller only needs 2.5 to $11 \%$ of its energy to excite the modes. 


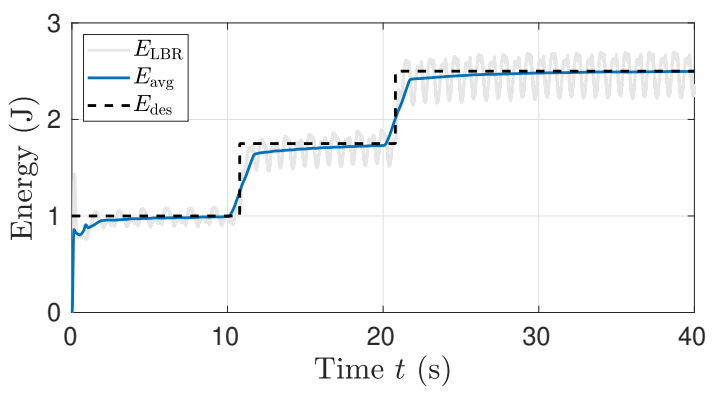

Fig. 12. This figure highlights the controller's ability to freely change the desired energy stepwise while converging to the desired energy level after a few seconds for 2 jumps of $0.75 J$ with a distance of $10 s$ each.

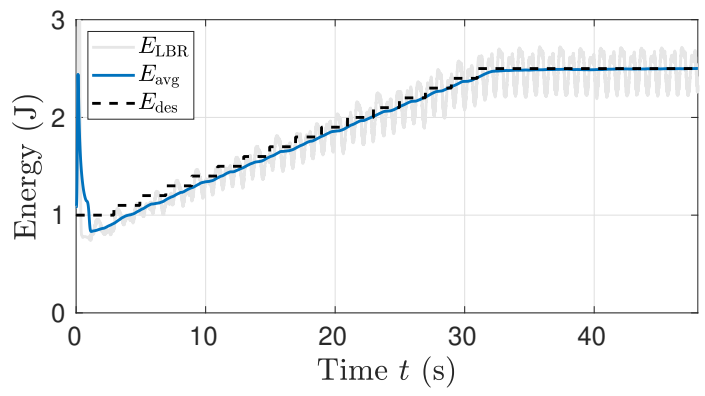

Fig. 13. This figure indicates the controller's ability to change the energy in a continuous way. Here, the energy increases every $2 s$ by $0.1 \mathrm{~J}$ up to a maximum of $2.5 \mathrm{~J}$

\section{CONClusion}

This paper showed that nonlinear modal theory could find robust implementation on highly articulated robotic platforms. A control architecture was proposed that excites nonlinear modal oscillations by simultaneously stabilizing eigenmanifolds and regulating an energy level. We design the novel controllers to be applicable in real-world scenarios. We thoroughly tested them in terms of efficiency, effectiveness, and robustness with multiple experiments performed with a KUKA iiwa with simulated elasticity. Future work will be devoted to extending these results to hybrid systems and applying nonlinear modes excitation to legged robots that could use their natural dynamics to move hyper-efficiently and robustly.

\section{REFERENCES}

[1] C. Della Santina, M. G. Catalano, and A. Bicchi, Soft Robots. Berlin, Heidelberg: Springer Berlin Heidelberg, 2020, ch. 1, pp. 1-15.

[2] L. Scalera, I. Palomba, E. Wehrle, A. Gasparetto, and R. Vidoni, "Natural motion for energy saving in robotic and mechatronic systems," Applied Sciences, vol. 9, no. 17, p. 3516, 2019.

[3] A. Albu-Schäffer, S. Wolf, O. Eiberger, S. Haddadin, F. Petit, and M. Chalon, "Dynamic modelling and control of variable stiffness actuators," in IEEE International Conference on Robotics and Automation, 2010, pp. 2155-2162.

[4] C. Della Santina, "Flexible manipulators," Encyclopedia of Robotics, 2021.

TABLE IV

ENERGY CONSUMPTION AND EFFICIENCY

\begin{tabular}{c|c|c|c|c|c|c|c} 
Mode & 1 & 2 & 3 & 4 & 5 & 6 & 7 \\
\hline Rigid $[J]$ & 19500 & 0 & 4419 & 2563 & 6797 & 3526 & 8489 \\
\hline Soft $[\mathrm{J}]$ & 1214 & 0 & 651.7 & 353.8 & 2986 & 932.3 & 2546 \\
\hline$E_{\text {soft }} / E_{\text {rigid }}$ & 0.062 & - & 0.15 & 0.14 & 0.44 & 0.26 & 0.30 \\
\hline$E_{\text {soft }} / E_{\text {tt }}$ & - & - & 0.11 & 0.024 & 0.054 & 0.062 & 0.070
\end{tabular}

[5] N. Kashiri, et al., "An overview on principles for energy efficient robot locomotion," Frontiers in Robotics and AI, vol. 5, no. 129, 2018.

[6] M. Calisti, G. Picardi, and C. Laschi, "Fundamentals of soft robot locomotion," Journal of The Royal Society Interface, vol. 14, no. 130, p. 20170101, 2017.

[7] J. P. Barreto, F.-F. Schöler, and B. Corves, "The concept of natural motion for pick and place operations," in New advances in mechanisms, mechanical transmissions and robotics. Springer, 2017, pp. 89-98.

[8] P. Boscariol, G. Boschetti, P. Gallina, and C. Passarini, "Spring design for motor torque reduction in articulated mechanisms," in International Conference on Robotics in Alpe-Adria Danube Region. Springer, 2017, pp. 557-564.

[9] G. Carabin, I. Palomba, E. Wehrle, and R. Vidoni, "Energy expenditure minimization for a delta-2 robot through a mixed approach," in European Congress on Computational Methods in Applied Sciences and Engineering. Springer, 2019, pp. 383-390.

[10] L. F. van der Spaa et al., "Unparameterized optimization of the spring characteristic of parallel elastic actuators," IEEE Robotics and Automation Letters, vol. 4, no. 2, pp. 854-861, 2019.

[11] L. B. Bonacchi, M. A. Roa, A. Sesselmann, F. Loeffl, A. AlbuSchaffer, and C. Della Santina, "Efficient and goal-directed oscillations in articulated soft robots: the point-to-point case," IEEE Robotics and Automation Letters, vol. 6, no. 2, pp. 2555-2562, 2021.

[12] A. Shiriaev, J. W. Perram, and C. Canudas-de Wit, "Constructive tool for orbital stabilization of underactuated nonlinear systems: Virtual constraints approach," IEEE Transactions on Automatic Control, vol. 50, no. 8, pp. 1164-1176, 2005.

[13] M. Maggiore and L. Consolini, "Virtual holonomic constraints for eulerlagrange systems," IEEE Transactions on Automatic Control, vol. 58, no. 4, pp. 1001-1008, 2012.

[14] G. Garofalo and C. Ott, "Passive energy-based control via energy tanks and release valve for limit cycle and compliance control," IFACPapersOnLine, vol. 51, no. 22, pp. 73-78, 2018.

[15] A. Banaszuk and J. Hauser, "Feedback linearization of transverse dynamics for periodic orbits," Systems \& control letters, vol. 26, no. 2, pp. 95-105, 1995.

[16] B. Yi et al., "Orbital stabilization of nonlinear systems via mexican sombrero energy shaping and pumping-and-damping injection," Automatica, vol. 112, p. 108661, 2020.

[17] C. F. Sætre, A. Shiriaev, L. Freidovich, S. V. Gusev, and L. Fridman, "Robust orbital stabilization: A floquet theory-based approach," arXiv preprint arXiv:2011.13674, 2020.

[18] R. Ortega et al., "Orbital stabilization of nonlinear systems via the immersion and invariance technique," International Journal of Robust and Nonlinear Control, vol. 30, no. 5, pp. 1850-1871, 2020.

[19] M. A. Hopkins et al., "Compliant locomotion using whole-body control and divergent component of motion tracking," in 2015 IEEE International Conference on Robotics and Automation (ICRA). IEEE, 2015, pp. $5726-5733$.

[20] M. Khoramshahi et al., "Adaptive natural oscillator to exploit natural dynamics for energy efficiency," Robotics and Autonomous Systems, vol. 97, pp. 51-60, 2017.

[21] C. Della Santina and A. Albu-Schaeffer, "Exciting efficient oscillations in nonlinear mechanical systems through eigenmanifold stabilization," IEEE Control Systems Letters, 2020.

[22] C. Della Santina, D. Calzolari, A. M. Giordano, and A. Albu-Schaffer, "Actuating eigenmanifolds of conservative mechanical systems via bounded or impulsive control actions," IEEE Robotics and Automation Letters, vol. 6, no. 2, pp. 2783-2790, 2021.

[23] A. Albu-Schaeffer and C. Della Santina, "A review on nonlinear modes in conservative mechanical systems," Annual Reviews in Control, 2020.

[24] A. Albu-Schaffer, D. Lakatos, and S. Stramigioli, "Strict nonlinear normal modes of systems characterized by scalar functions on riemannian manifolds," IEEE Robotics and Automation Letters, 2021.

[25] G. Kerschen, M. Peeters, J.-C. Golinval, and A. F. Vakakis, "Nonlinear normal modes, part i: A useful framework for the structural dynamicist," Mechanical systems and signal processing, vol. 23, no. 1, pp. 170-194, 2009.

[26] C. Della Santina, D. Lakatos, A. Bicchi, and A. Albu-Schäffer, "Using nonlinear normal modes for execution of efficient cyclic motions in articulated soft robots," Proc. Int. Symp. on Experimental Robotics ISER, 2020.

[27] J. H. Friedman, J. L. Bentley, and R. A. Finkel, "An algorithm for finding best matches in logarithmic expected time," ACM Transactions on Mathematical Software (TOMS), vol. 3, no. 3, pp. 209-226, 1977.

[28] S. Gudmundsson and E. Kappos, "On the geometry of tangent bundles," Expositiones Mathematicae, vol. 20, no. 1, pp. 1-41, 2002.

[29] S. Virga and M. Esposito, "Iiwa stack," https://github.com/IFL-CAMP/ iiwa_stack 2016, [Online; accessed 12-July-2021]. 